\title{
Deep optical observations of the massive galaxy cluster Abell 1413*
}

\author{
D. Castagné ${ }^{1,2,5}$, G. Soucail ${ }^{1,3}$, E. Pointecouteau ${ }^{1,2}$, A. Cappi $^{4,5}$, S. Maurogordato ${ }^{4}$, C. Benoist ${ }^{4}$, and C. Ferrari ${ }^{4}$ \\ ${ }^{1}$ Université de Toulouse, UPS-Observatoire Midi-Pyrénées, IRAP, 31400 Toulouse, France \\ e-mail: genevieve.soucail@irap.omp.eu \\ 2 CNRS, Institut de Recherche en Astrophysique et Planétologie, 9 Avenue du Colonel Roche, BP 44346, 31028 Toulouse Cedex 4 , \\ France \\ 3 CNRS, Institut de Recherche en Astrophysique et Planétologie, 14 Avenue Edouard Belin, 31400 Toulouse, France \\ ${ }^{4}$ Laboratoire Lagrange, UMR 7293, Université de Nice Sophia-Antipolis, CNRS, Observatoire de la Côte d'Azur, 06300 Nice, \\ France \\ 5 INAF - Osservatorio Astronomico di Bologna, via Ranzani 1, 40127 Bologna, Italy
}

Received 5 March 2012 / Accepted 27 September 2012

\begin{abstract}
Aims. We investigate the morphology and dynamics of the massive nearby galaxy cluster Abell 1413.

Methods. Using wide field imaging and spectroscopic data obtained from observations at the Canada-France-Hawaii Telescope and archival SDSS data, we build a sample of more than 250 galaxies covering a region centered on the cluster and extending to its outskirts on large scales.

Results. We probe the cluster morphology analyzing the projected density distribution of galaxies and find a strong variation in the ellipticity of the distribution, from 0.8 at the center of the cluster down to $\sim 0.35$ at the periphery. This may be the tracer of a prolate global mass distribution, slightly attenuated in the collisional distribution of the gas. We also study the large scale environment, identifying several structures associated to the cluster and organized along the major axis in the North-South direction. From spectroscopic data we derive the velocity dispersion and we investigate the overall dynamics of the cluster. The regular velocity dispersion supports the case for a prolate cluster aligned in the plane of the sky and embedded in a larger scale structure.
\end{abstract}

Key words. galaxies: clusters: general - galaxies: clusters: individual: Abell 1413 - galaxies: distances and redshifts

\section{Introduction}

Galaxy clusters are the most massive gravitationally bound structures in the Universe. As representative of the high end of the mass function, they are key ingredients in the definition of the cosmological paradigm (Eke et al. 1996; Bahcall et al. 1997). According to the standard cosmological framework, they assembled by accretion of matter within deep potential wells, and via a succession of merger events and relaxation phases. This hierarchical scenario of structure formation is strongly supported by extensive numerical simulations (Navarro et al. 1997; Evrard et al. 2002).

However, observations show that clusters of galaxies are complex systems made of different components, with departures from spherical symmetry and the presence of substructures in the galaxy density distribution (Dressler \& Shectman 1988; Girardi et al. 1997; Aguerri \& Sánchez-Janssen 2010), and dynamical disturbances apparent in the galaxy velocity distribution, which can be the consequence of group/cluster mergers as expected in the history of massive systems (e.g., Ferrari et al. 2005; Maurogordato et al. 2008). Moreover galaxies, like dark matter can be considered as collisionless particles. Their spatial distribution is therefore more sensitive to substructures and anisotropies in the mass distribution, while X-ray emission, because of the collisional nature of the hot baryonic gas, is much

\footnotetext{
* Appendix $\mathrm{A}$ is available in electronic form at http: //www . aanda.org
}

smoother and regular (e.g., Jones \& Forman 1999; Schuecker et al. 2001; Lopes et al. 2006). The galaxy distribution is also a better tracer of the large scale environment of massive clusters, which are embedded in a neighbouring filamentary network, while the low sensitivity of X-ray observations to the warm intracluster gas precludes any deep insight into the large scale distribution of the gas.

Within this framework, in this paper we focus on a detailed case study of the nearby cluster Abell $1413(z=0.1412)$. This is a well known rich cluster, dominated by a giant $\mathrm{cD}$ galaxy which displays a strong X-ray emission (Pratt \& Arnaud 2002; Pointecouteau et al. 2005; Vikhlinin et al. 2006; Hoshino et al. 2010; Ettori et al. 2010). Despite the high ellipticity of its X-ray morphology $(\epsilon \simeq 0.3)$, it is often considered as a relaxed and virialized cluster. However, a recent detection of a radio diffuse emission (Govoni et al. 2009), although at a low significance level, could be interpreted as a sign for recent merger activity. Moreover, its extremely large cD galaxy has an outstanding extended halo with very high ellipticity. Even if it is well known that the ellipticity of $\mathrm{cD}$ galaxies increases with radius, the $\mathrm{cD}$ in A 1413 is an extreme example of this effect (Porter et al. 1991; Gonzalez et al. 2005).

To clarify the morphological and dynamical structure of this cluster at different scales, we perform an analysis of the galaxy distribution on a large field centered on the cluster, using both multi-color imaging and spectroscopic data. In Sects. 2 and 3 the different data sets used for the analysis, as well as the selection criteria defined to identify cluster galaxies, are 
described. Section 4 is devoted to the study of the morphology of Abell 1413 via the galaxy density maps. The analysis of the cluster velocity distribution and of the cluster luminosity function are presented respectively in Sects. 5 and 6. The cluster total mass and mass-to-light ratio estimates are discussed in Sect. 7. Concluding remarks are provided in Sect. 8.

Throughout this paper we adopt the following values for the cosmological parameters: $H_{0}=70 \mathrm{~km} \mathrm{~s}^{-1} \mathrm{Mpc}^{-1}, \Omega_{\mathrm{M}}=0.3$ and $\Omega_{\Lambda}=0.7$. In this case $1^{\prime}$ corresponds to $149 \mathrm{kpc}$ at the cluster redshift.

\section{The data}

\subsection{Photometric catalogs}

A 1413 was observed with the Canada-France-Hawaii Telescope (CFHT) and its wide field imager MegaCam on February 20, 2007 (run ID 2007AH96) and March 18, 2007 (run ID 2007AH97, PI: G. Morrison). Imaging data were retrieved from the CADC archives ${ }^{1}$ and consist in two images taken in the $R$ and $Z$ bands. We benefited from the MegaCam image stacking pipeline MegaPipe to obtain directly the co-added images photometrically calibrated (Gwyn 2008). Total exposure times are respectively 1600 and $1920 \mathrm{~s}$ in $R$ and $Z$ and the average seeing is $0.81^{\prime \prime}$ and $1.12^{\prime \prime}$. Both images are centered at the position of the cluster central galaxy: $\alpha=11^{\mathrm{h}} 55^{\mathrm{m}} 18^{\mathrm{s}}$ and $\delta=23^{\circ} 24^{\prime} 18^{\prime \prime}$.

Objects were measured with SExtractor in dual-mode (Bertin \& Arnouts 1996) with the $R$-band image as the reference one. 132599 objects were extracted, with completeness magnitudes $R \simeq 25.0$ and $Z \simeq 24.5$. Several SExtractor parameters were used to separate galaxies from stars. First, all objects with a $C L A S S \_S T A R$ larger than 0.9 were considered as stars and removed. Second, a $\mu_{\max }$-Magnitude diagram allowed us to remove saturated objects, cosmic rays and defects (points "below" the star line). Finally, a total of 102031 objects were selected as "galaxies". Throughout this study the magnitude MAG_AUTO will be considered as the reference magnitude.

To complement the photometric data with other passbands and to apply a membership selection based on photometric redshifts, photometric data from the SDSS DR $7^{2}$ were also retrieved (Adelman-McCarthy 2009). Although SDSS data are shallower than our CFHT data, we were able to extract 21455 objects with magnitudes in the 5 photometric bands $(u, g, r, i$ and $z)$ within a field of 1.4 square degree centered on the central galaxy of Abell 1413, and we performed a cross-identification between the SDSS objects and the $R$-band catalog. The final catalog includes all galaxies with a photometric redshift estimated from the SDSS photometry (Csabai et al. 2003). It contains more than 15000 galaxies with CFHT $R, Z$ and SDSS $u, g, r, i, z$ magnitudes.

\subsection{Spectroscopic data}

Spectroscopic data in the field of A 1413 come from two independent datasets. The first sample was obtained with spectroscopic data obtained at CFHT with the MOS instrument (RunID 1997AF13, PI: Maurogordato). MOS is a low resolution multiobject spectrograph with a field of view of $9.7 \times 14.5 \mathrm{arcmin}^{2}$.

\footnotetext{
1 The Canadian Astronomy Data Centre is operated by the National Research Council of Canada with the support of the Canadian Space Agency.

2 Photometric redshifts were retrieved through the CASJOB interface: http://casjobs.sdss.org/casjobs/
}

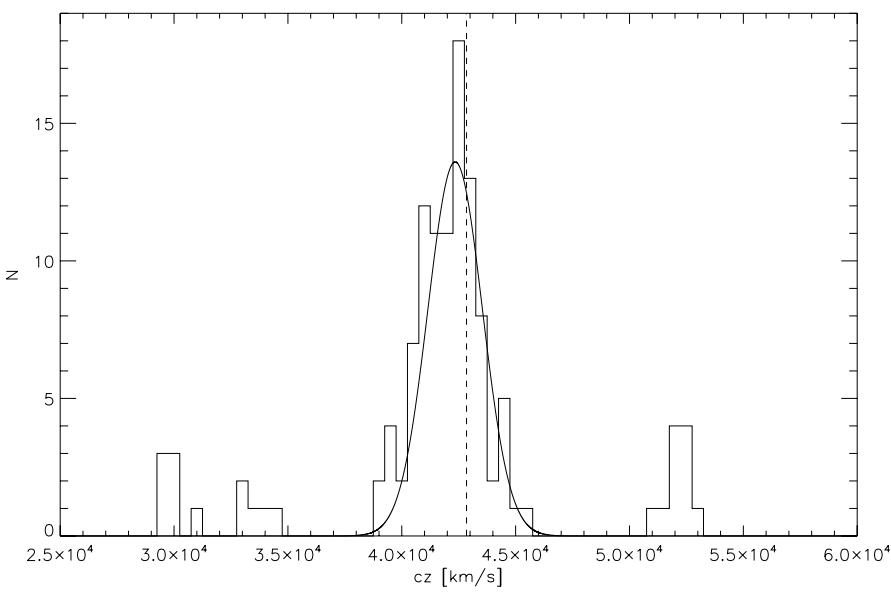

Fig. 1. Radial velocity histogram of galaxies within a $15^{\prime}$ radius centered on A 1413, in bins of $500 \mathrm{~km} \mathrm{~s}^{-1}$. The solid line represents the best Gaussian fit from ROSTAT; the dashed line indicates the velocity of the central BCG.

We used the grism O300, providing a dispersion of $5 \AA$ pixel and a resolution $R \sim 400$. A pass-band filter, isolating the wavelength range 4000-6200 $\AA$ and covering the main spectral features used to measure the redshift of cluster members (from [OII] emission line to $\mathrm{Mg}$ absorption lines) was designed on purpose. It allowed us to put several slits along the dispersion axis without superimposing the spectra on the CCD. This provided a gain of a factor 2 to 3 in efficiency for multi-object spectroscopy. After data reduction and redshift measurement, we ended with a spectroscopic catalog of 107 galaxies (see Table A.1 in Appendix). Data are also available in electronic form at CDS.

Additional spectroscopic data were retrieved from the Sloan Digital Sky Survey DR7 (Abazajian et al. 2009). 162 objects were found in the field of view covered by MegaCam data with an accurate redshift measurement $\left(\delta z\right.$ better than $\left.10^{-3}\right)$. Among them, 158 were considered as galaxies in the SExtractor classification. We identified 14 galaxies in common with our CFHT data. Their redshifts are consistent within the errors, with an average difference $\Delta z \sim 3 \times 10^{-4}$.

The final joint spectroscopic catalog contains 256 galaxies with a measured redshift. It is added to the photometric catalog; if available, the spectroscopic redshift supersedes the photometric redshift. We estimate that within the central field corresponding to the MOS field of view we reach a completeness better than $90 \%$ up to $R \sim 17.5$.

\section{Cluster membership}

\subsection{Spectroscopic selection}

We first limited our spectroscopic galaxy sample to objects located within a $15^{\prime}$ radius from the cluster center. This value is roughly representative of the virial radius, as measured for example by Pratt \& Arnaud (2002) with X-rays data: $R_{200}=1676 \mathrm{kpc}$ in physical units, i.e. an angular extension of $11.1^{\prime}$. After $3 \sigma$ clipping, a total of 97 galaxies were retained as A 1413 members within our spectroscopic catalog. Through ROSTAT (Beers et al. 1990), we found the mean velocity $C_{\mathrm{BI}}=42345 \pm 128 \mathrm{~km} \mathrm{~s}^{-1}$ $(z=0.1412 \pm 0.0004)$ and velocity dispersion $S_{\mathrm{BI}}=1196 \pm$ $89 \mathrm{~km} \mathrm{~s}^{-1}$. Figure 1 shows the velocity distribution histogram. 


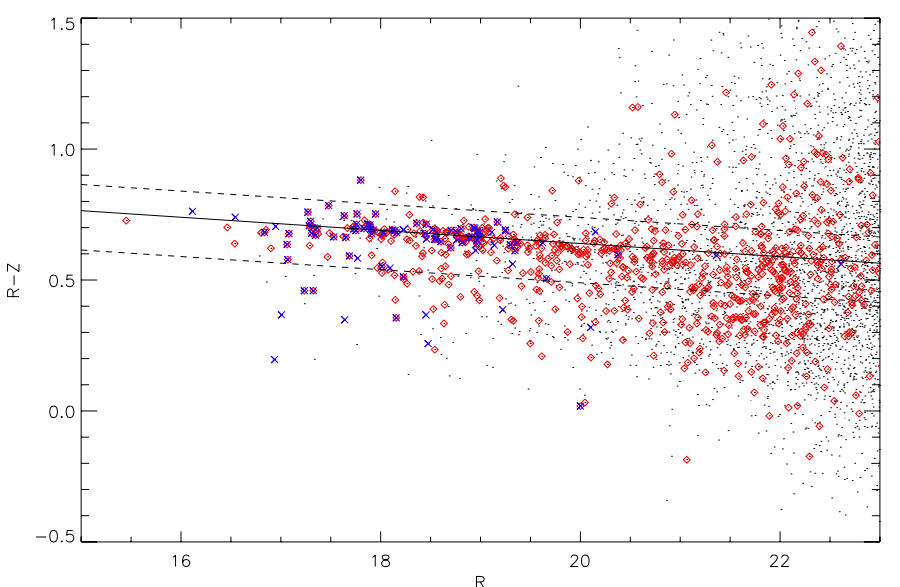

Fig. 2. Color-magnitude diagram of the galaxies in the field of Abell 1413 and within a radius of $15^{\prime}$. The red diamonds are cluster members defined according to their photometric redshift while blue crosses are spectroscopically confirmed cluster members. The two dashed lines delimit the locus of the galaxies considered as early-type galaxies.

\subsection{Photometric selection in the color-magnitude diagram}

It is well known that early-type galaxies in clusters settle along a well defined sequence in a color-magnitude diagram (Visvanathan \& Sandage 1977). This so-called "red-sequence" (RS) can be explained assuming that early-type galaxies had an intitial single burst of star formation and followed passive evolution. The slope of the RS represents the mass-metallicity relation: more massive galaxies are dominated by metal-enriched stars which have a reddened spectral energy distribution. The presence of the RS makes possible to identify different galaxy populations (Metcalfe et al. 1994): (i) galaxies on the RS are early-types; (ii) the "blue" zone under the RS is mainly populated by late-type galaxies with a foreground and background contamination; (iii) in the area above the RS there are galaxies outside the cluster, preferentially in the background (Thompson \& Gregory 1993; Biviano et al. 1995; López-Cruz et al. 2004).

The RS for A 1413 is plotted in Fig. 2 in a color-magnitude diagram built with the CFHT R and Z catalogs. The fit of the RS is done using confirmed galaxy members in the spectroscopic catalog. From a linear regression of the RS function, we find a slope $a=-0.025$ and we adopt a range of \pm 0.15 around the RS to select early-type galaxies in the photometric catalog. To be consistent with previous spectroscopic sample and to avoid too much background contamination we limited our sample to galaxies with $R<21$ and within a radius of $15^{\prime}$ from the BCG. From the 1017 objects initially selected, 548 belong to the RS and are considered as early-type galaxies.

\subsection{Cluster member selection with photometric redshifts}

Thanks to the well-calibrated and very homogeneous photometric data of the SDSS, photometric redshifts were computed and adjusted to fit the spectroscopic data. Photo- $z$ applied to SDSS data are described in Csabai et al. (2003). The authors emphasize the difficulty to state generic errors on photo- $z$, as they depend on the object redshift and magnitude. However, within the redshift range of A 1413, we can refer to their confidence interval which varies from $\delta z \simeq 0.03$ for red galaxies to $\delta z \simeq 0.09$ for blue ones. In the following, we adopt an average value of $\delta z=0.05$ fairly representing the average SDSS photo- $z$ uncertainty. Our sample is again limited to $R<21$, because we
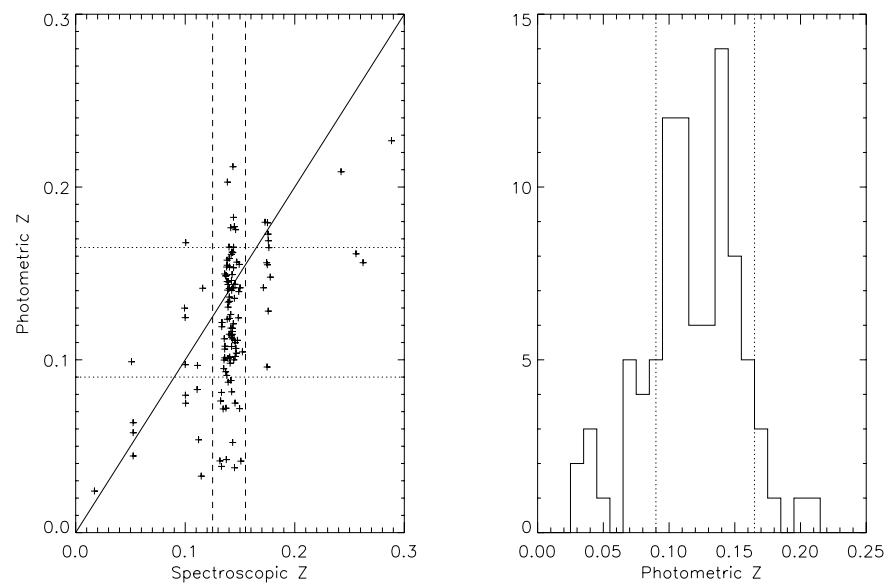

Fig.3. Left: comparison between spectroscopic redshifts (CFHT+SDSS) and photometric redshifts from the SDSS DR7 $(R<21)$. Dashed line represents the spectroscopic range used to select cluster members [0.125-0.155]. Dotted lines show the photometric range chosen to define cluster membership [0.09, 0.165]. Right: photometric redshifts histogram of the spectroscopically confirmed cluster members $(0.125<z<0.155)$ with magnitude $R<21$. Dotted line show the photometric range defining cluster membership $[0.09$, $0.165]$.

Table 1. Number of galaxies located in a radius of $15^{\prime}$ from the center and considered as cluster members according to the different selection criteria defined in the text.

\begin{tabular}{ccccc}
\hline \hline Selection & $R<18$ & $R<19$ & $R<20$ & $R<21$ \\
\hline S1 & 76 & 244 & 503 & 1017 \\
S2 & 61 & 184 & 339 & 548 \\
S3 & 47 & 147 & 243 & 359 \\
\hline
\end{tabular}

consider that at fainter levels the accuracy in the photometric redshift decreases and more outliers will contaminate the galaxy sample (Adelman-McCarthy 2009).

Figure 3 compares the SDSS photometric redshifts and the spectroscopic ones for the galaxies in our spectroscopic catalog. There is a good agreement between the data, with the usual dispersion in photometric redshifts (Csabai et al. 2003; Wen et al. 2009). From the photometric redshift histogram built with secured galaxy members, all objects with a photometric redshift between 0.09 and 0.165 were considered as "cluster members". We finally merged the spectroscopic and photometric samples and ended up with 359 clusters members within $15^{\prime}$ and with $R<21$.

In summary, we end up with three selection criteria which will be used to derive the density maps of galaxies:

S1 encompasses all objects selected as galaxies in the $R$-band catalog within a distance $<15^{\prime}$ from the cluster center;

$\mathrm{S} 2$ is obtained from the RS selection and contains galaxies found within the aforementioned limits around the red sequence best fit line;

S3 corresponds to a redshift selection of cluster members with either a spectroscopic redshift or a photometric redshift within the range $0.09<z<0.165$.

Statistics for the various selections are shown in Table 1. 

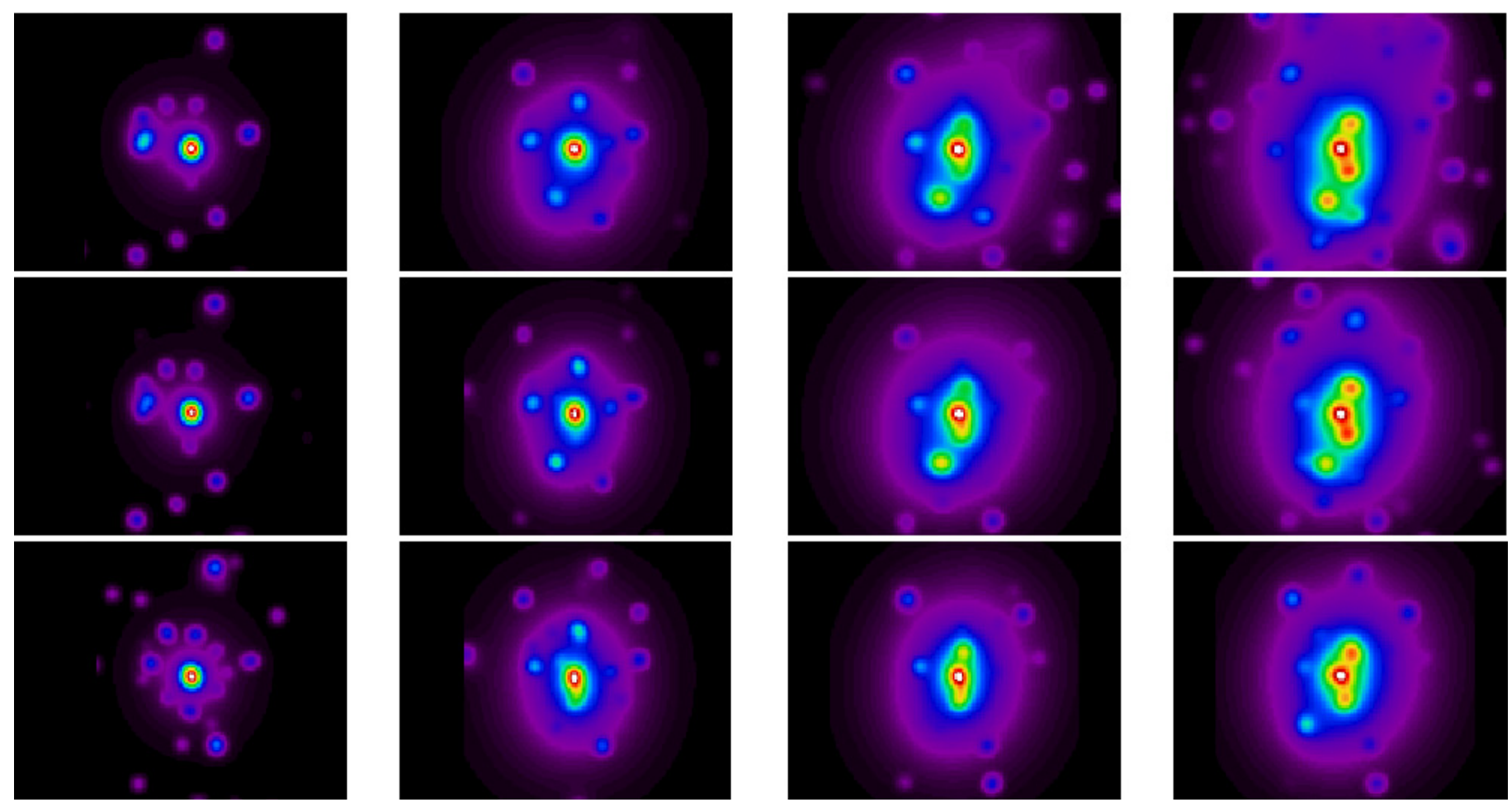

Fig. 4. Projected galaxy density maps for different magnitude cuts in the $R$-band (from left to right: $R<18, R<19, R<20, R<21$ ) and different galaxy selections: S1, S2 and S3 (from top to bottom) as defined in the text and Table 1 . The FOV is $29 \times 24 \operatorname{arcmin}^{2}$ (or equivalently $\left.4.3 \times 3.6 \mathrm{Mpc}^{2}\right)$.
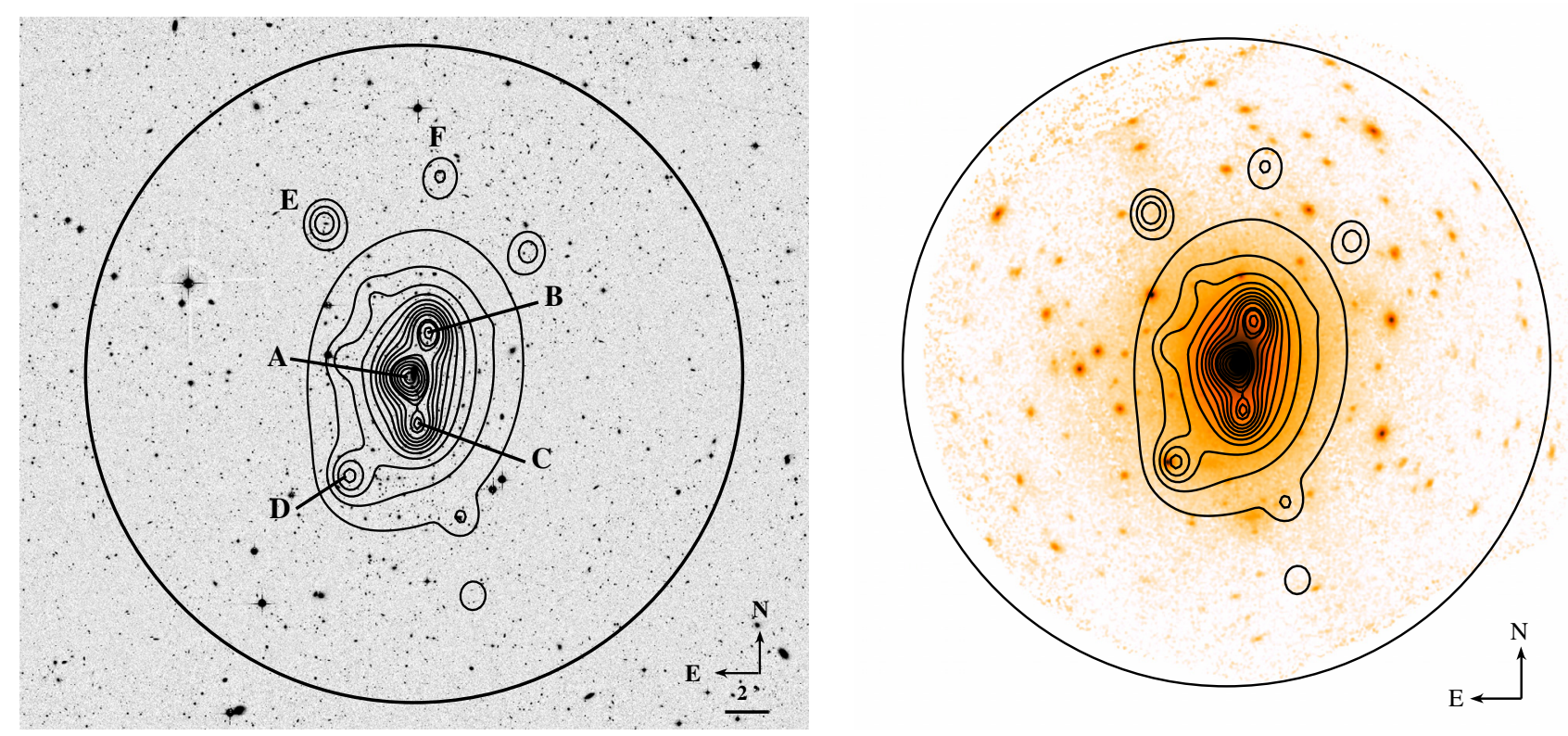

Fig. 5. Left: contours of the density map of photometrically selected galaxy members $(R<21$ and criterion S3) overlaid on the CFHT-R image. Substructures are identified with capital letters. The black circle represents the radius $R=15^{\prime}$ (or equivalently $2.2 \mathrm{Mpc}$ at the cluster redshift). Right: same contours overlaid on the XMM-Newton image.

\section{Density maps and morphology of Abell 1413}

\subsection{Projected density maps}

To study the morphology of A 1413, we computed the galaxy density maps using a 2D generalization of the algorithm described in Fadda et al. (1998) - see also Ferrari et al. (2005). Density maps were computed applying various cuts in magnitude and with the 3 selection criteria described in the previous section; they are shown in Fig. 4. The population of early-type galaxies in clusters is a good tracer of the overall galaxy distribution within the potential well (Biviano et al. 2006), so the selection $\mathrm{S} 2$ is well representative of the overall cluster morphology. Selection S3 based on photo- $z$ criteria allows us to decontaminate the density maps from spurious structures possibly due to background or foreground clumps or projection artifacts. It also includes red and blue galaxies and may be sensitive to bluer clumps of galaxies. Therefore for our aims the last map obtained 
D. Castagné et al.: Deep optical observations of the massive galaxy cluster Abell 1413

Table 2. Characteristics of the different substructures found in A 1413.

\begin{tabular}{cccc}
\hline \hline & $\begin{array}{c}\text { RA } \\
(J 2000)\end{array}$ & $\begin{array}{c}\text { Dec } \\
(\text { J2000) }\end{array}$ & $\begin{array}{c}d^{a} \\
(\operatorname{arcmin})\end{array}$ \\
\hline $\mathrm{A}$ & 115518 & +232410 & 0.3 \\
$\mathrm{~B}$ & 115515 & +232610 & 2.0 \\
$\mathrm{C}$ & 115517 & +232200 & 2.3 \\
$\mathrm{D}$ & 115530 & +231940 & 5.5 \\
$\mathrm{E}$ & 115536 & +233110 & 8.2 \\
$\mathrm{~F}$ & 115513 & +233320 & 9.1 \\
\hline
\end{tabular}

Notes. ${ }^{(a)}$ Distance to the cluster center.

with selection S3 and $R<21$ (or equivalently $M_{R} \sim M^{\star}+3$, see Sect. 6) is the most useful and will be used in the rest of the paper.

Isodensity maps reveal a large scale elliptical structure populated with clumps at smaller scales as expected from a hierarchical scenario of structure formation (White \& Frenk 1991). They are labeled in the overlay of the density contours on the CFHT-R image (Fig. 5). The three major clumps (A-C) show up in the center of A 1413 and are aligned approximately in the North-South direction, as the cluster main elliptical axis. Three other substructures D, E and F show up at slightly larger distances from the central BCG, in the North-East and the SouthEast directions. To check the reality of the structures, we considered only those which are detected on both density maps built with criteria S2 and S3. Only 266 galaxies are common to the two samples (about $2 / 3$ of the galaxies for $\mathrm{S} 3$ and less than $50 \%$ for S2). We also checked that reducing the redshift interval for the selection $\mathrm{S} 3$, these structures are still detected. In practice, the clump E marginally fulfills these criteria. On the contrary, D and $\mathrm{F}$ remain even if they have a rather diffuse shape. All other structures seen in the maps are not statistically significant. The characteristics of the 6 confirmed clumps are given in Table 2.

In Fig. 5, we also overlaid the XMM-Newton image obtained from the $30 \mathrm{ks}$ observation (guaranteed time) performed in Dec. 2000 (OBSID\#0112230501) and published in Pratt \& Arnaud (2002). Whereas these authors used only the EMOS $1 \& 2$ cameras, we produced a count rate image in $0.3-2 \mathrm{keV}$ energy band from the three XMM-Newton cameras (i.e. EMOS $1 \& 2$ and EPN). To do so we reprocessed the X-ray data in a similar way as presented in Pratt \& Arnaud (2002), and in further work by these authors (Pointecouteau et al. 2004; Pratt et al. 2007). A particle background derived from a compilation of Filter Wheel Closed observations was subtracted from our image (Croston et al. 2008). This image is further used in the next section to investigate the cluster ellipticity. But none of the substructures identified here are correlated with any X-ray enhancement above the strong cluster X-ray flux. They most probably correspond to low mass groups or substructures which are less relaxed than the overall baryonic distribution traced by the collisional intracluster gas.

\subsection{Ellipticity}

We analyzed the cluster elliptical shape on the basis of the galaxy density map built with the S3 sample and on the XMM-Newton data. In both optical and X-ray cases, the image was fitted within increasing apertures starting from $1^{\prime}$ up to $13^{\prime}$. A 2 D elliptical King profile (King 1966) was fitted in the galaxy density map, whereas the fit on the X-ray image was performed with a 2D elliptical $\beta$-model (Cavaliere \& Fusco-Femiano 1976; Pratt \& Arnaud 2002). The ellipticity is defined as $\epsilon=1-r_{\mathrm{b}} / r_{\mathrm{a}}$, where $r_{\mathrm{a}}$

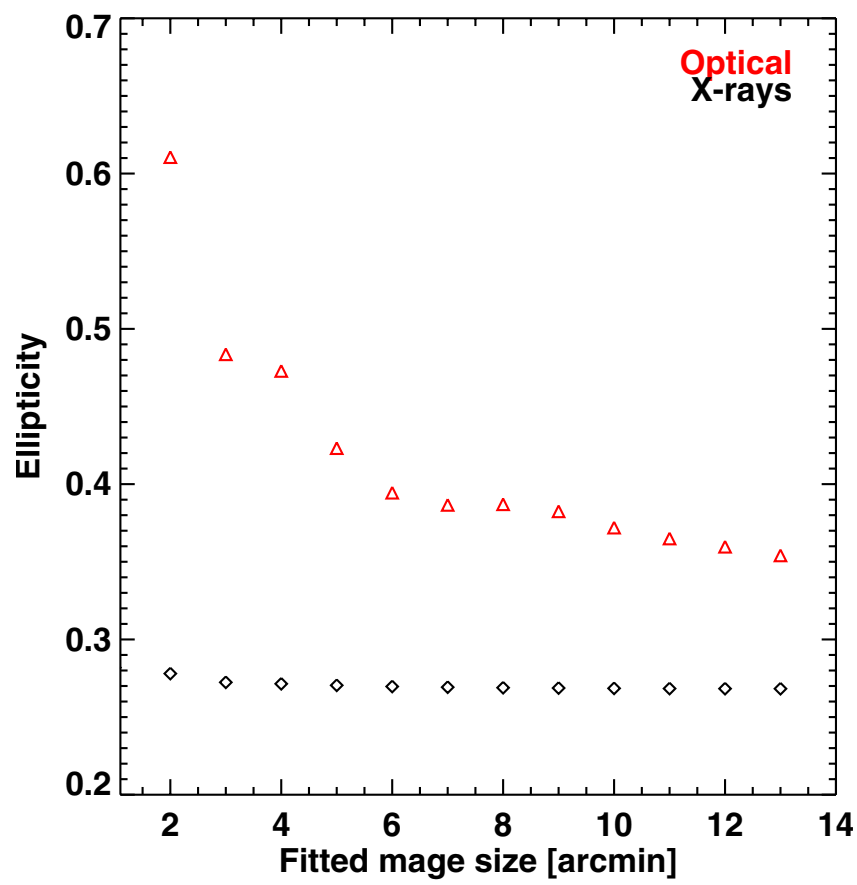

Fig. 6. Comparison of the ellipticity of A 1413 fitted over increasing angular apertures and derived from the galaxy density map (red triangles) and from the XMM-Newton image (black diamond).

and $r_{\mathrm{b}}$ are respectively the core radius of the 2D King $/ \beta$-profiles along the major and the minor axis. The evolution of $\epsilon$ with increasing aperture is reported in Fig. 6.

In the fit of the XMM-Newton image, the values of $\epsilon$ quickly converge toward 0.27 , which is fully compatible with the global value of 0.29 derived by Pratt \& Arnaud (2002) over the radial range $\left[3^{\prime}-13^{\prime}\right]$. From the galaxy density map, the convergence is slower and it reaches a value $\epsilon \sim 0.35$, which is much larger than the ellipticity of the gas distribution. This is not surprising if we consider that the collisional gas relaxes more quickly within the cluster potential than the non-collisional galaxies and reaches more rapidly a spherically symmetric distribution; however, Abell 1413 seems a rather extreme example of this effect.

More surprisingly, the ellipticity derived from the optical density maps drastically increases toward the center of the cluster, up to $\sim 0.7$ at the center. This strong increase in $\epsilon$ can be explained by the clumpiness of the central region of A 1413 which is extremely elongated along the North-South axis. It also matches the results presented by Feldmeier et al. (2002) on the ellipticity of the central $\mathrm{cD}$ galaxy and its extended envelope, i.e. $\epsilon=0.8$ (Fig. 7). The authors emphasize the rare occurrence of such extreme elliptical shape for bright central galaxies (i.e., average $\epsilon \sim 0.4$ ) and they note that A 1413 is the most extreme example. It is worth mentioning that they derived their estimate from the light emission of the $\mathrm{cD}$ galaxy and its surroundings, whereas our derivation down to the very center of A 1413 was obtained from the galaxy number density distribution, and one could expect the latter to be less elliptical than the former one.

The best fit values for the position angle, $\theta$ (i.e. the angle between the major axis of the elliptical model and the North direction, counted clockwise) range between 0 and -10 degrees over the fitted apertures. This interval encompasses the best fit value found from the XMM-Newton data, i.e. $\theta \sim-2$ degrees. It is also in good agreement with the average value of $\sim-5$ degrees found for the central cD galaxy by Feldmeier et al. (2002). The major orientation angle and the unambiguous ellipticity of 


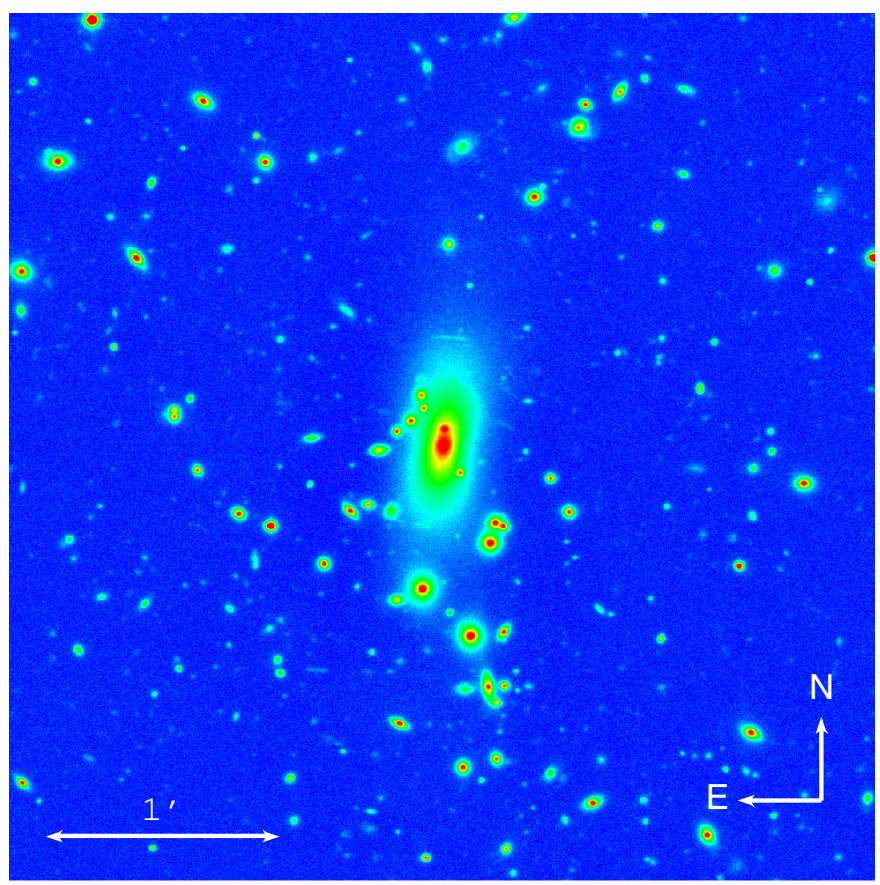

Fig. 7. Detailed view of the cD galaxy at the center of Abell 1413. The extended envelope covers more than $100 \mathrm{kpc}$ along the major axis, with an ellipticity as high as 0.7 .

A 1413 along the North-South direction from the very central parts to the outskirts of the cluster are good probes, together with the very regular velocity dispersion (see next Sect. 5), of the prolate nature and orientation of A 1413 in the plane of the sky. This preferred direction may also relate to the structure around the cluster and a privileged accretion direction, as discussed in the following section.

\subsection{The large scale environment of Abell 1413}

To study the large scale environment of A 1413, we have analyzed the galaxy density distribution within a 4 sq. degr. region centered on A 1413. At the cluster redshift, this covers an area $\sim 20 h^{-1} \mathrm{Mpc} \times 20 h^{-1} \mathrm{Mpc}$. For this purpose, the SDSS photometric and spectroscopic data were used with the same selection in photometric redshift $0.09<z<0.165$ and built a density map of the selected galaxies (Fig. 8).

The NASA Extragalactic Database ${ }^{3}$ was also queried for clusters in the vicinity of A 1413 and within the same redshift range. 15 structures are part of the Northern Sky Cluster (NCS) catalog (Gal et al. 2003) and its extended version (Lopes et al. 2004), or are identified from the SDSS (Wen et al. 2009; Hao et al. 2010) and the MaxBCG (Koester et al. 2007) clusters. The redshifts are based on photometric properties of the galaxies or on photometric redshifts, so we selected all structures identified within a range [0.1:0.18] well centered on the redshift of Abell 1413. These structures are presented in Table 3 and overlaid on the density map (Fig. 8).

The cluster $\mathrm{ZwCl} 1154.2+2435$ is the most significant overdensity detected in this area; its significance is confirmed by its X-ray diffuse emission which was detected by ROSAT Bohringer et al. (2000). We selected the galaxies spectroscopically confirmed (using the SDSS DR7 data) in a circle of 5 arcmin radius around this cluster. Analysis of the velocity

\footnotetext{
http://nedwww.ipac.caltech.edu/
}

distribution with ROSTAT gives a location of $C_{\mathrm{BI}}=42279 \pm$ $315 \mathrm{~km} \mathrm{~s}^{-1}(z=0.1409)$ and a velocity dispersion of $S_{\mathrm{BI}}=$ $912 \pm 200 \mathrm{~km} \mathrm{~s}^{-1}$.

The galaxy density map extends mainly to the North and the South of A 1413, without significant extension in the East or West directions. Several groups/clusters are located within the $15^{\prime}$ radius around the cluster center. Combined with a few more distant clusters in the Northern extension, they trace three Y-shaped directions (Fig. 8). Interestingly, $\mathrm{ZwCl} 1154.2+2435$ lies on the privileged direction defined at smaller scales by A 1413/NSCS J115531+233447/MaxBCG $\mathrm{J} 179.01253+23.89451$, and at a similar redshift. Extended X-ray emission is also detected in this cluster from ROSAT data Bohringer et al. (2000). Probably A 1413 lies at the heart of a North-South elongated super-structure, and it is still accreting components along these directions.

Finally we note that there is a well defined cluster, 20' west of A 1413 but with a photometric redshift estimate below the redshift range of the selected galaxies (NSC J115657+232322, $z \simeq 0.058$ ). The presence of a strong concentration of galaxies is confirmed in our CFHT image, and the redshift of the brightest galaxy is $z=0.1007$ in the SDSS database; if this is the redshift of the system, there is no physical connexion with A 1413.

\section{Cluster kinematics}

\subsection{Velocity distribution of the cluster}

We analyzed the spectroscopic catalog previously obtained by combining redshifts successfully obtained from our CFHT/MOS data with those retrieved from the SDSS/DR7, in the 1 square degree region covered by the CFHT/Megacam imaging (256 redshifts). After a first blind run of ROSTAT on the whole distribution, we selected galaxies with velocities within a wide window of $\pm 5000 \mathrm{~km} \mathrm{~s}^{-1}$ around our first estimate of the central location to start the dynamical analysis of the cluster (119 objects). A critical issue for the analysis of the velocity distribution of the cluster is to correctly assess the cluster membership of the galaxies. In this case we did not limit to a radius of $15^{\prime}$, as in Sect. 3.1, but adopted another criterium for eliminating interlopers (hence the slightly different numbers). For all cluster members candidates we plotted the velocity offset with respect to the central velocity $(\Delta v)$, as a function of the angular distance $\theta$ to the cluster center, defined by the position of the BCG (Fig. 9). Clusters members are expected to be located in the $(\Delta v, \theta)$ plane within a caustic with a large width at small radius, decreasing at large ones. At the light of this plot, we eliminated two obvious interlopers at $\left(\Delta v \simeq 4000 \mathrm{~km} \mathrm{~s}^{-1}, \theta \simeq 30^{\prime}\right)$.

The final catalog of 117 objects was analyzed with ROSTAT (Beers et al. 1990). This sample meets the criterion of maximum velocity gap of $1000 \mathrm{~km} \mathrm{~s}^{-1}$ between adjacent members in velocity space, and are considered as our "reference sample" in the dynamical analysis. The mean velocity and velocity dispersion of the cluster obtained with the bi-weight method are $C_{\mathrm{BI}}=42387 \pm 111 \mathrm{~km} \mathrm{~s}^{-1}(z=0.14129 \pm 0.0004)$ and $S_{\mathrm{BI}}=$ $1196 \pm 83 \mathrm{~km} \mathrm{~s}^{-1}$. These values are very stable with respect to the method used (bi-weight, mean, $3 \sigma$ clipping). We analyzed separately two sub-samples corresponding to our data from CFHT and to the data retrieved from SDSS/DR7 respectively, and obtained comparable values for the cluster mean velocity and velocity dispersion. Several tests were applied to our reference sample, in order to check if the velocity distribution is consistent with one or more Gaussian functions. The 15 normality tests provided by ROSTAT do not reject the Gaussian hypothesis at a significance level $<20 \%$. Furthermore, we computed shape 


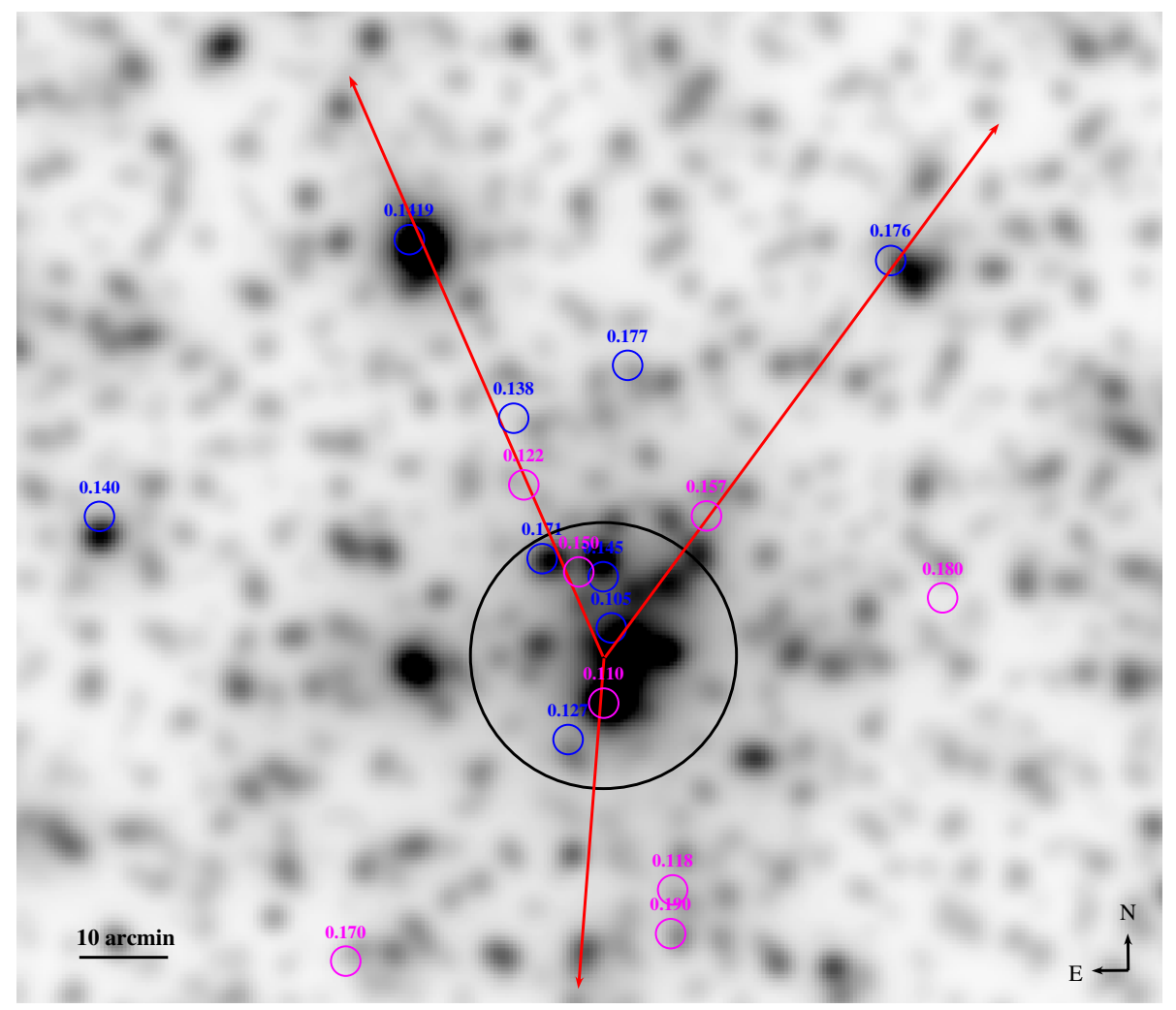

Fig. 8. Galaxy density map of objects with magnitude $R<21$ and photometric redshift $0.09<z<0.165$ (S3-like criterion) in a $2 \times 2$ sq. degrees field centered on Abell 1413 . Blue circles represent clusters in the FOV and with a photometric redshift $0.1<z<0.18$ while other circles in magenta are identified by their redshift estimate only. The black circle marks $R_{200}=15^{\prime}$. Red lines indicated possible mains axis of accretion in A 1413 surroundings. estimators such as skewness and kurtosis and the Asymmetry Index (AI) and Tail Index (TI) introduced by Bird (1993) to study the asymmetry and flatness of a distribution. From our reference sample, we derived values of skewness and kurtosis of -0.179 and 2.876 respectively, and asymmetry and tail indexes of $A I=-0.382$ and $T I=0.971$. Significance levels for the four shape estimators were estimated from Table 2 of Bird (1993), and again do not reject the Gaussian hypothesis at a significance level $<20 \%$.

The velocity of the central $\mathrm{cD}$ galaxy has been measured by Humason et al. (1956) $v_{\mathrm{BCG}}=42844 \pm 100 \mathrm{~km} \mathrm{~s}^{-1}$, and more recently refined in the Catalog of Principal Galaxies (Paturel et al. 1989) to $v_{\mathrm{BCG}}=42832 \pm 71 \mathrm{~km} \mathrm{~s}^{-1}$. From our previous analysis, this implies a velocity offset of $450 \pm 210 \mathrm{~km} \mathrm{~s}^{-1}$ compared to the mean cluster velocity, which is $2 \sigma$ significant. Velocity offsets of BCGs are often found in clusters far from equilibrium, for instance when undergoing major merging events. If confirmed this BCG velocity offset would suggest that A 1413 may be in a non-fully relaxed dynamical state.

\subsection{Subclustering kinematic tests indicator}

Among the various tests probing cluster substructure, we selected the 3D $\Delta$-test developed by Dressler \& Shectman (1988). For each cluster galaxy, the ten closest neighbours are considered. The cumulative differences between their mean velocity and velocity dispersion and those of the overall cluster are computed into a $\delta$ value associated with the considered cluster galaxy. A map can be drawn from these $\delta$ values, where each galaxy is represented with a circle proportional to $\delta$. Thus, large circles represent high spatial and kinematic differences with the global cluster properties. Finally a global $\Delta$ value is obtained as the sum of all $\delta$. A $\Delta$ value close to the number of galaxies in the sample will argue in favor of a cluster without substructures and kinematic distortion. We applied this 3D $\Delta$-test

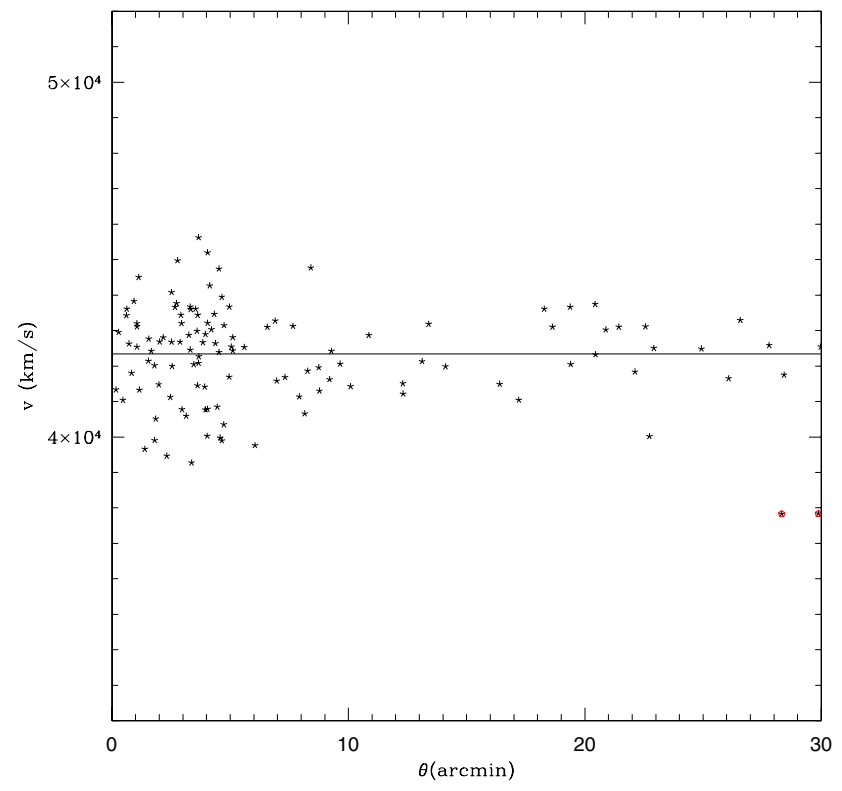

Fig. 9. Radial velocity as a function of the distance to the cluster center. The solid line represents the mean velocity estimated by ROSTAT. The two objects in red circles with $V<38000 \mathrm{~km} \mathrm{~s}^{-1}$ and $\theta>28$ arcmin are considered as interlopers as they lie far away from the general caustic embedding the cluster members in the velocity-distance plane. They will be excluded in the following analysis.

on the 97 spectroscopically confirmed cluster members according to the selection described in Sect. 3.1 (Fig. 10), finding a global value of $\Delta=101.6$, very close to the number of galaxies used in the test (97 galaxies); we did not detect any significant concentration of large circles, which would have indicated the presence of significant substructure. 
Table 3. Characteristics of the large scale structures identified around A 1413.

\begin{tabular}{lcccc}
\hline \hline Name & $\begin{array}{c}\text { RA } \\
(\mathrm{J} 2000)\end{array}$ & $\begin{array}{c}\text { Dec } \\
(\mathrm{J} 2000)\end{array}$ & Redshift & $\begin{array}{c}\text { Distance } \\
(\operatorname{arcmin})\end{array}$ \\
\hline GMBCG J178.81296+23.46510 & $11: 55: 15$ & $23: 27: 54$ & $0.105(\mathrm{p})$ & 3.5 \\
NSCS J115519+231840 & $11: 55: 19$ & $23: 18: 40$ & $0.110(\mathrm{e})$ & 5.6 \\
GMBCG J178.78964+23.57010 & $11: 55: 09$ & $23: 34: 12$ & $0.145(\mathrm{p})$ & 9.9 \\
NSCS J115531+233447 & $11: 55: 31$ & $23: 34: 47$ & $0.150(\mathrm{e})$ & 10.9 \\
MaxBCG J178.90194+23.23649 & $11: 55: 36$ & $23: 14: 11$ & $0.127(\mathrm{p})$ & 11.0 \\
WHL J115549.4+233626 & $11: 55: 49$ & $23: 36: 26$ & 0.171 & 13.8 \\
NSC J115428+234140 & $11: 54: 28$ & $23: 41: 40$ & $0.157(\mathrm{p})$ & 20.8 \\
NSC J115557+234526 & $11: 55: 58$ & $23: 45: 26$ & $0.122(\mathrm{p})$ & 23.0 \\
NSC J115444+225542 & $11: 54: 45$ & $22: 55: 42$ & $0.118(\mathrm{p})$ & 29.7 \\
MaxBCG J179.01253+23.89451 & $11: 56: 03$ & $23: 53: 40$ & $0.138(\mathrm{p})$ & 31.1 \\
GMBCG J178.77945+24.00328 & $11: 55: 07$ & $24: 00: 12$ & $0.177(\mathrm{p})$ & 35.8 \\
NSCS J115725+224653 & $11: 57: 25$ & $22: 46: 53$ & $0.170(\mathrm{e})$ & 47.5 \\
ZwCl 1154.2+2435 & $11: 56: 55$ & $24: 15: 36$ & 0.1419 & 55.7 \\
GMBCG J178.23881+24.21734 & $11: 52: 57$ & $24: 13: 02$ & $0.176(\mathrm{p})$ & 58.3 \\
MaxBCG J179.86190+23.68976 & $11: 59: 27$ & $23: 41: 23$ & $0.140(\mathrm{p})$ & 59.3 \\
\hline
\end{tabular}

Notes. The redshifts are either photometric redshifts (p) (Koester et al. 2007; Wen et al. 2009; Hao et al. 2010) or redshift estimates (e) (Gal et al. 2003; Lopes et al. 2004), except for ZwCl 1154.2+2435 (also referenced as MaxBCG J179.23236+24.25997) which redshift was measured by Allen et al. (1992). The distance refers to the central cD galaxy in A 1413. Note that at the cluster redshift, $1^{\prime}$ scales as $150 h^{-1} \mathrm{kpc}$.

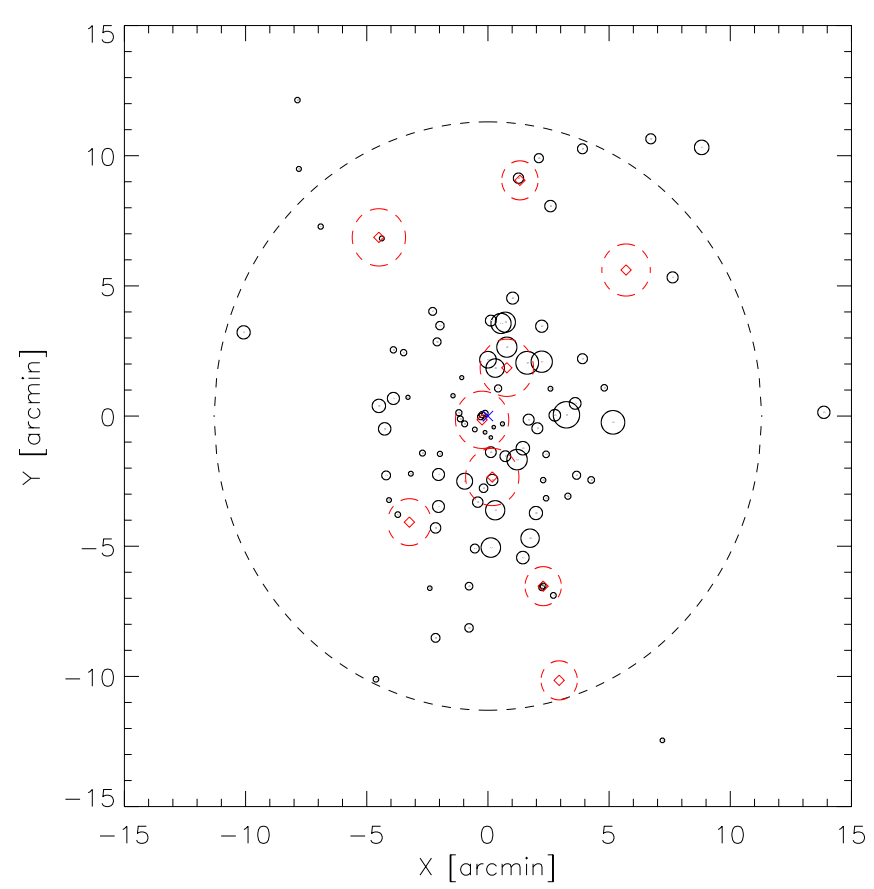

Fig. 10. Projected positions of the galaxies in our spectroscopic catalog $(R<21)$, represented by circles with sizes weighted by the $\delta$ estimator of Dressler \& Shectman (1988). Concentration of large circles indicate a correlated spatial and kinematic variation. The substructures identified in the galaxy density map are shown as red diamonds delimited by dashed circles.

Finally, we computed the significance level of our results using bootstrap techniques and 1000 Monte Carlo simulations. We found a significance level of $67 \%$, whereas subclustering is confirmed for significance levels lower than $10 \%$. In summary, A 1413 is confirmed as a very regular and dynamically relaxed system despite its highly elongated shape. The few sub-halos detected by the morphological study appear to be normal clumps hosted by such a halo, as expected in a hierarchical formation of structures.

\section{The luminosity function of A 1413}

The number of galaxies per unit of luminosity and volume defines the luminosity function (LF) of a cluster. In a hierarchical framework, the total luminosity encompassed within a given radius is linked to the number of galaxies and to the mass enclosed in the same radius. Moreover, the luminosity density profile is a good tracer of the cluster mass (Katgert et al. 2004). Luminosity functions are generally fitted with a Schechter function (Schechter 1976), which combines a bright end exponential shape and a faint end power law. For galaxies selected according to their photometric redshift (selection S3), we converted $R$-band magnitudes into absolute magnitudes assuming a distance modulus $\mu=39.12$, a galactic extinction of 0.01 , and a $K$-correction of 0.37 (which was computed for an elliptical-type spectral energy distribution with the Bruzual and Charlot code, Bruzual \& Charlot 2003).

We binned objects into 0.5 mag intervals up to an absolute magnitude of -18.5 , corresponding to our completeness limit $R=21.0$. We fitted a Schechter function as shown in Fig. 11 . Our best fit parameters are: $N^{\star}=260 \pm 45 \mathrm{gal} / \mathrm{mag} / \mathrm{deg}^{2}$, $M^{\star}=-21.8 \pm 0.2$ and $\alpha=-0.8 \pm 0.1$, with a reduced $\chi^{2}=0.57$. The value of $M^{\star}$ is rather close to the value found by Barkhouse et al. (2007) $\left(M^{*}=-22.36\right.$, obtained with a fixed the faint end slope $\alpha=-1$ ) and is typical for rich clusters. The faint end slope is found slightly larger than the canonical value $\alpha=-1$ but it has a large uncertainty associated to the background corrections in our sample S3. As a test case, we also generated a sub-sample $\mathrm{S} 4$ by introducing the same red-sequence cut as in the sample S2, but in the S3 sample, selecting 266 objects. We fitted the luminosity function for these galaxies which now should represent the early-type galaxy population, finding respectively: $N^{\star}=216 \pm 40 \mathrm{gal} / \mathrm{mag} / \mathrm{deg}^{2}, M^{\star}=-21.7 \pm 0.2$ and $\alpha=-0.7 \pm 0.1$, with a reduced $\chi^{2}=0.64$. The values of $M^{\star}$ and $\alpha$ are consistent with the estimates for selection S3. Finally, we integrated the luminosity function to derive the total cluster luminosity in the $R$-band: $L_{\mathrm{tot}}=9.1 \pm 3.2 \times 10^{12} L_{\odot}$ while the integrated luminosity of the early-type galaxies is $L_{\mathrm{RS}}=6.7 \pm 2.7 \times 10^{12} L_{\odot}$. 


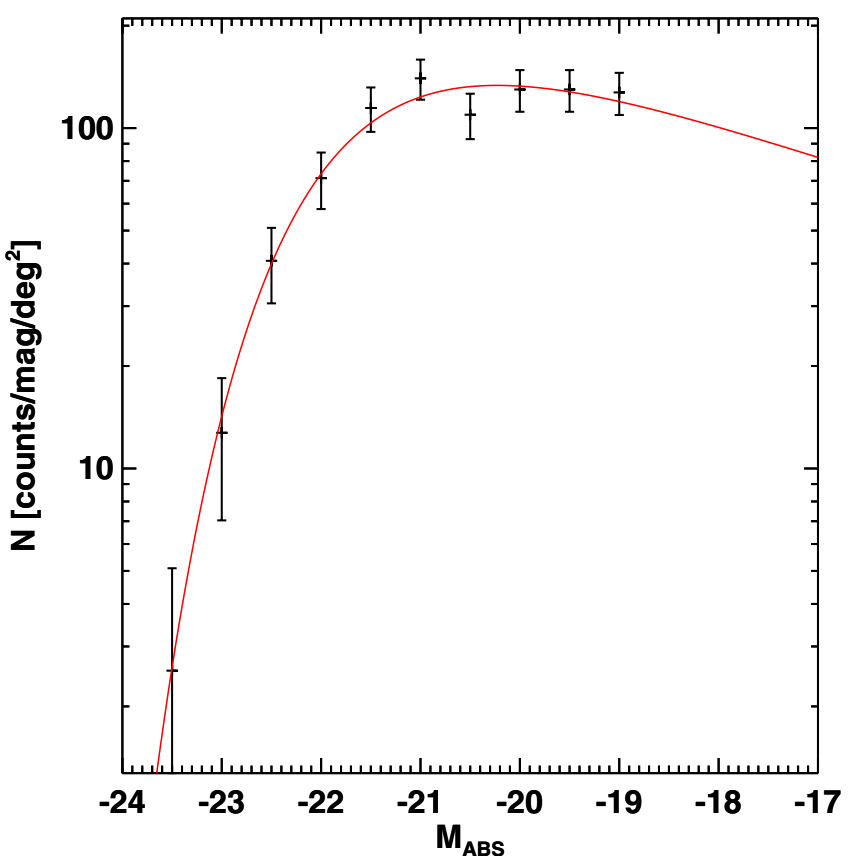

Fig. 11. A 1413 luminosity function in the $R$ band for galaxies selected by their photometric redshift and within a $15^{\prime}$ radius around the cluster center. The solid red line show the best fit luminosity function.

\section{The mass of the cluster A 1413}

Making use of our optical data, we followed a standard approach to derive the virial mass of the cluster (see for instance Ferrari et al. 2005; Maurogordato et al. 2008). Applying the virial theorem seems reasonable given the aussian shape of the velocity distribution (Fig. 1). Moreover our velocity dispersion of $\sigma=1196 \pm 83 \mathrm{~km} \mathrm{~s}^{-1}$ compares well to the one derived from the $\sigma-T$ relation $\left(\sigma=1018 \mathrm{~km} \mathrm{~s}^{-1}\right.$, Lubin \& Bahcall 1993), assuming an X-ray temperature of $k T=6.5 \mathrm{keV}$ (Pratt \& Arnaud 2002). On the other hand, the cluster shape is far from spherical and we will see how this can affect our results.

We briefly recall the formalism of a virial mass estimation from a population of cluster galaxies:

$$
\begin{aligned}
& M_{\mathrm{vir}}=\frac{3}{2} \frac{\pi}{G} R_{\mathrm{vir}} \sigma_{r}^{2} \\
& R_{\mathrm{vir}}=\frac{2 N}{N-1} R_{\mathrm{h}} \\
& R_{\mathrm{h}}=\frac{N(N-1)}{2}\left(\sum_{i<j}^{N-1} \sum_{j=i+1}^{N} \frac{1}{R_{\mathrm{ij}}}\right)
\end{aligned}
$$

where $R_{\mathrm{vir}}$ is the projected virial radius deduced from the projected harmonic radius $R_{\mathrm{h}}$ and the virial radius is $r_{\mathrm{vir}}=(\pi / 2) R_{\mathrm{vir}}$. $R_{\mathrm{ij}}$ is the projected distance between the $i$ th and the $j$ th galaxies for a total number of $N$ objects in the system.

To estimate the harmonic radius, we used the galaxies in the S3 selection $\left(0.09<z_{\text {phot }}<0.165\right.$ and $\left.R<21\right)$, applying both the pairwise and the ring-wise methods (see Eqs. (2) and (3) in Carlberg et al. 1996). We performed the estimate by cutting our catalog at different radii (between 10 and 30 arcmin), finding the ring-wise harmonic radius systematically larger than the pairwise radius by a factor $\sim 1.7$. As discussed by Carlberg et al. (1996), the larger value of the ring-wise harmonic radius is a systematic effect due to cluster ellipticity and subclustering: for their cluster sample they estimated a mean correction factor of 1.28. The larger factor 1.7 for A 1413 can be due both to its significant ellipticity and also to the fact that the pairwise harmonic radius may be underestimated. We therefore will give and discuss both estimates, cutting the catalog at $15^{\prime}$ from the cluster center. This radius is large enough to include most of the cluster galaxies (and mass) on the basis of the galaxy and gas density maps.

From Eq. (1), we derived with the pairwise estimate a projected virial radius of $R_{\mathrm{vir}}=1.0 \pm 0.1 \mathrm{Mpc}$, and a virial mass $M_{\text {vir }}=1.5 \pm 0.3 \times 10^{15} M_{\odot}$, while with the ring-wise estimate $R_{\mathrm{vir}}=1.7 \pm 0.1 \mathrm{Mpc}$ and $M_{\mathrm{vir}}=2.4 \pm 0.3 \times 10^{15} M_{\odot}$.

We define the quantity $\Delta_{\text {vir }} \equiv \bar{\rho}_{\text {vir }} / \rho_{\mathrm{c}}$ where $\bar{\rho}_{\text {vir }} \equiv$ $M_{\text {vir }} /\left(4 \pi r_{\text {vir }}^{3}\right)$, following Carlberg et al. (1996). If the previous estimate of $r_{\text {vir }}$ is correct we expect $\Delta_{\text {vir }} \sim 200$, and we should find nearly the total mass of the cluster within $r_{\mathrm{vir}}$. In practice, $M_{\text {vir }}$ is not fully included within $r_{\text {vir }}$, so $\Delta_{\text {vir }}$ for a given $r_{\text {vir }}$ may give an overestimate of the true value. For the pairwise virial radius we find $\Delta_{\mathrm{vir}} \sim 510$, and our value of $r_{\mathrm{vir}}$ is therefore consistent with the value $r_{500}=1.13 \mathrm{Mpc}$ found by Pointecouteau et al. (2005). On the other hand, for the ring-wise virial radius we find $\Delta_{\text {vir }} \sim 190$, and our value of $r_{\text {vir }}$ is also consistent with the X-ray observations which have provided a radius $R_{200} \simeq 1.7 \mathrm{Mpc}$ : with XMM-Newton (Pratt \& Arnaud 2002; Pointecouteau et al. 2005), Chandra (Vikhlinin et al. 2006; Ettori et al. 2010) and Suzaku (Hoshino et al. 2010). However, $\mathrm{X}$-ray observations give mass measurements falling in the range $0.6<M_{200}<0.9 \times 10^{15} M_{\odot}$, i.e. a factor $2.7-4$ smaller than our optical estimates. This factor is too large to be explained by the fact that at $R_{\text {vir }}$ we estimate the total mass and not the mass within $R_{\text {vir }}$ X-ray measurements are derived under the hypothesis of hydrostatic equilibrium, and numerical simulations have shown that under this hypothesis and with the use of a spectroscopically derived temperature $\mathrm{X}$-ray mass estimates tend to be underestimated by 10-15\% (Kay et al. 2004; Piffaretti \& Valdarnini 2008). In principle, the very high ellipticity could significantly affect these estimates. Knowing that the error in the mass profile measurement in X-rays is mainly driven by the errors in the temperature profile, Pratt \& Arnaud (2002) have shown the consistency of temperature profiles extracted in elliptical and circular concentric annuli. However, the exceptional high value of the ellipticity of the dark matter distribution could induce larger discrepancies for A 1413 than for more regular clusters.

Finally, using the cluster total luminosity estimated in Sect. 6, we derive a mass-to-light ratio for A 1413 of $M / L=$ $264 \pm 64 h_{70} M_{\odot} / L_{\odot}$. This value is consistent with the value extrapolated from the $L_{\mathrm{op}}-M_{200}$ relation of Popesso et al. (2007): using our mass estimate we find an expected mass-to-light ratio $M / L=251 \pm 83 h_{70} M_{\odot} / L_{\odot}$.

\section{Conclusions}

We have conducted a thorough optical analysis of the nearby cluster of galaxies Abell 1413. On the basis of X-ray observations this rich system is considered a relaxed system, despite its highly elliptical shape (Pratt \& Arnaud 2002; Vikhlinin et al. 2006). With the present work, based on deep CFHT observations combined with archive SDSS data, we have investigated the optical morphology of this cluster from its central region including the cD galaxy to its large scale environment.

From the galaxy number density distribution, we estimated the value of the cluster ellipticity $\epsilon \sim 0.35$ at large radii, 
increasing up to $\epsilon \sim 0.8$ at the cluster center (i.e. $r<1^{\prime}$ ). The elongation of the cluster is approximately aligned in the North-South direction, a few degrees Westwards. The central cD galaxy also aligns along this axis and its extended envelope shows a coherent high elongation of the light distribution (Feldmeier et al. 2002). In comparison, the ellipticity study from the $X M M$-Newton observation gives a systematic smaller value $\epsilon \sim 0.28$ whatever the aperture radius. We did not find any sign of tilt with increasing radius for the cluster main axis which follows the North-South axis. In the galaxy distribution we also detected 3 well identified substructures within the very center of the cluster $\left(r<3^{\prime}\right)$. These clumps are again aligned along the North-South direction and contribute to the main cluster ellipticity. All these characteristics point toward a coherent view of a very elongated mass distribution in the cluster, in particular for the non-collisional component, i.e. the galaxies. The collisional nature of the gas may have generated a more regular distribution with a shorter relaxation time than the galaxies. A direct mapping of the dark matter distribution using the weak lensing effect would re-enforce this statement if the high ellipticity of the reconstructed map is confirmed. However, the velocity distribution of A 1413 is not significantly different from a Gaussian distribution, characteristic of a relaxed state. This is rather surprising in comparison with the statement of a non-fully relaxed cluster presented above, and this could be indicative of a very prolate shape of A 1413 in the plane of the sky. We also remind that the spectroscopic sample is still incomplete, especially in the outer parts of the cluster. In the large scale environment of Abell 1413 we detected also several privileged directions traced by various groups and small clusters mainly located along the North and South directions. This strenghtens the case for a main North-South accretion axis, which is feeding A 1413, the node of the local super-structure environment.

From our optical observations, we derived a total luminosity of early-type galaxies within a radius of $R_{200}: L_{200} \simeq 9.5 \pm 3.3 \times$ $10^{12} L_{\odot}$. A dynamical analysis of our spectroscopic sample gives a virial mass $M_{200}=2.4 \pm 0.3 \times 10^{15} M_{\odot}$. Finally the mass-tolight ratio in the cluster is $M / L=264 \pm 64$ in solar units. These global quantities are in agreement with the expected properties for nearby massive clusters (Popesso et al. 2007). However, we note that our mass estimate is significantly higher than the average mass estimates derived from X-ray observations by at least a factor of 2 , even if it remains marginally compatible, at the $2 \sigma$ level. Again, a weak lensing analysis of this cluster could allow us to have a different point of view to study the mass distribution in the cluster and its surroundings. It could also provide a complementary mass estimate which hopefully would fix the uncertainties pointed out in this study. In addition, we will present the analysis of a deep XMM-Newton observation of A 1413 in a forthcoming paper in order to assess the mass distribution down to the outskirts of the clusters and characterise the thermodynamical state of these external regions.

Acknowledgements. D.C. acknowledges Lauro Moscardini for support during his stay in Bologna. The authors are indebted toward Roser Pello for fruitful discussions in particular on the use of the publicly available code Hyper- $z$ and to Johan Richard for his share of spectroscopic data for the central cD galaxy. This work is based on observations obtained with MegaPrime/MegaCam, a joint project of Canada-France-Hawaii Telescope (CFHT) and CEA/DAPNIA, at the Canada-France-Hawaii Telescope (CFHT) which is operated by the National Research Council (NRC) of Canada, the Institut National des Sciences de l'Univers of the Centre National de la Recherche Scientifique (CNRS) of France and the University of Hawaii. This research has made use of the NASA/IPAC Extragalactic Database (NED) which is operated by the Jet
Propulsion Laboratory, California Institute of Technology, under contract with the National Aeronautics and Space Administration, and of the SIMBAD database, operated at CDS, Strasbourg, France (http://simbad.u-strasbg. $\mathrm{fr} / \mathrm{simbad} /$ ), and of archival data from the SDSS project (http://www. sdss. org). D.C. acknowledges the financial support of the VINCI program of the Franco-Italian University.

\section{References}

Abazajian, K. N., Adelman-McCarthy, J. K., Agüeros, M. A., et al. 2009, ApJS, 182,543

Adelman-McCarthy, J. K. E. 2009, VizieR Online Data Catalog, 2294, 0

Aguerri, J. A. L., \& Sánchez-Janssen, R. 2010, A\&A, 521, A28

Allen, S. W., Edge, A. C., Fabian, A. C., et al. 1992, MNRAS, 259, 67

Bahcall, N. A., Fan, X., \& Cen, R. 1997, ApJ, 485, L53

Barkhouse, W. A., Yee, H. K. C., \& López-Cruz, O. 2007, ApJ, 671, 1471

Beers, T. C., Flynn, K., \& Gebhardt, K. 1990, AJ, 100, 32

Bertin, E., \& Arnouts, S. 1996, A\&AS, 117, 393

Bird, C. M. 1993, PASP, 105, 1495

Biviano, A., Durret, F., Gerbal, D., et al. 1995, A\&A, 297, 610

Biviano, A., Murante, G., Borgani, S., et al. 2006, A\&A, 456, 23

Bohringer, H., Voges, W., Huchra, J. P., et al. 2000, ApJS, 129, 435

Bruzual, G., \& Charlot, S. 2003, MNRAS, 344, 1000

Carlberg, R. G., Yee, H. K. C., Ellingson, E., et al. 1996, ApJ, 462, 32

Cavaliere, A., \& Fusco-Femiano, R. 1976, A\&A, 49, 137

Colless, M. 1989, MNRAS, 237, 799

Croston, J. H., Pratt, G. W., Böhringer, H., et al. 2008, A\&A, 487, 431

Csabai, I., Budavári, T., Connolly, A. J., et al. 2003, AJ, 125, 580

Dressler, A., \& Shectman, S. A. 1988, AJ, 95, 985

Eke, V. R., Cole, S., \& Frenk, C. S. 1996, MNRAS, 282, 263

Ettori, S., Gastaldello, F., Leccardi, A., et al. 2010, A\&A, 524, A68; Erratum: 2011, A\&A, 526, C1

Evrard, A. E., MacFarland, T. J., Couchman, H. M. P., et al. 2002, ApJ, 573, 7

Fadda, D., Slezak, E., \& Bijaoui, A. 1998, A\&AS, 127, 335

Feldmeier, J. J., Mihos, J. C., Morrison, H. L., Rodney, S. A., \& Harding, P. 2002, ApJ, 575, 779

Ferrari, C., Benoist, C., Maurogordato, S., Cappi, A., \& Slezak, E. 2005, A\&A, 430, 19

Gal, R. R., de Carvalho, R. R., Lopes, P. A. A., et al. 2003, AJ, 125, 2064

Girardi, M., Escalera, E., Fadda, D., et al. 1997, ApJ, 482, 41

Gonzalez, A. H., Zabludoff, A. I., \& Zaritsky, D. 2005, ApJ, 618, 195

Govoni, F., Murgia, M., Markevitch, M., et al. 2009, A\&A, 499, 371

Gwyn, S. D. J. 2008, PASP, 120, 212

Hao, J., McKay, T. A., Koester, B. P., et al. 2010, ApJS, 191, 254

Hoshino, A., Patrick Henry, J., Sato, K., et al. 2010, PASJ, 62, 371

Humason, M. L., Mayall, N. U., \& Sandage, A. R. 1956, AJ, 61, 97

Jones, C., \& Forman, W. 1999, ApJ, 511, 65

Katgert, P., Biviano, A., \& Mazure, A. 2004, ApJ, 600, 657

Kay, S. T., Thomas, P. A., Jenkins, A., \& Pearce, F. R. 2004, MNRAS, 355, 1091

King, I. R. 1966, AJ, 71, 64

Koester, B. P., McKay, T. A., Annis, J., et al. 2007, ApJ, 660, 239

Lopes, P. A. A., de Carvalho, R. R., Capelato, H. V., et al. 2006, ApJ, 648, 209

Lopes, P. A. A., de Carvalho, R. R., Gal, R. R., et al. 2004, AJ, 128, 1017

López-Cruz, O., Barkhouse, W. A., \& Yee, H. K. C. 2004, ApJ, 614, 679

Lubin, L. M., \& Bahcall, N. A. 1993, ApJ, 415, L17

Maurogordato, S., Cappi, A., Ferrari, C., et al. 2008, A\&A, 481, 593

Metcalfe, N., Godwin, J. G., \& Peach, J. V. 1994, MNRAS, 267, 431

Navarro, J. F., Frenk, C. S., \& White, S. D. M. 1997, ApJ, 490, 493

Paturel, G., Fouque, P., Bottinelli, L., \& Gouguenheim, L. 1989, A\&AS, 80, 299

Piffaretti, R., \& Valdarnini, R. 2008, A\&A, 491, 71

Pointecouteau, E., Arnaud, M., Kaastra, J., \& de Plaa, J. 2004, A\&A, 423, 33

Pointecouteau, E., Arnaud, M., \& Pratt, G. W. 2005, A\&A, 435, 1

Popesso, P., Biviano, A., Böhringer, H., \& Romaniello, M. 2007, A\&A, 464, 451

Porter, A. C., Schneider, D. P., \& Hoessel, J. G. 1991, AJ, 101, 1561

Pratt, G. W., \& Arnaud, M. 2002, A\&A, 394, 375

Pratt, G. W., Böhringer, H., Croston, J. H., et al. 2007, A\&A, 461, 71

Schechter, P. 1976, ApJ, 203, 297

Schuecker, P., Böhringer, H., Reiprich, T. H., \& Feretti, L. 2001, A\&A, 378, 408

Thompson, L. A., \& Gregory, S. A. 1993, AJ, 106, 2197

Tonry, J., \& Davis, M. 1979, AJ, 84, 1511

Vikhlinin, A., Kravtsov, A., Forman, W., et al. 2006, ApJ, 640, 691

Visvanathan, N., \& Sandage, A. 1977, ApJ, 216, 214

Wen, Z. L., Han, J. L., \& Liu, F. S. 2009, ApJS, 183, 197

White, S. D. M., \& Frenk, C. S. 1991, ApJ, 379, 52

Pages 11 to 12 are available in the electronic edition of the journal at http://www . aanda. org 


\section{Appendix A: Spectroscopic data obtained at CFHT}

Table A.1. List of redshifts measured at CFHT with the MOS instrument.

\begin{tabular}{|c|c|c|c|c|c|c|}
\hline ID & $\begin{array}{c}\text { RA } \\
\text { J2000 }\end{array}$ & $\begin{array}{c}\text { Dec } \\
\text { J2000 }\end{array}$ & $\begin{array}{c}\text { Redshift } \\
z\end{array}$ & $\begin{array}{l}\text { Velocity } \\
\left(\mathrm{km} \mathrm{s}^{-1}\right)\end{array}$ & $\begin{array}{c}\text { Error } \\
\left(\mathrm{km} \mathrm{s}^{-1}\right)\end{array}$ & $R$ \\
\hline 1 & $11: 55: 34.7$ & $23: 22: 01.4$ & 0.1362 & 40846 & 68 & 4.57 \\
\hline 2 & $11: 55: 33.5$ & $23: 26: 50.8$ & 0.1422 & 42638 & 43 & 8.07 \\
\hline 3 & $11: 55: 31.3$ & $23: 25: 03.1$ & 0.1354 & 40591 & 43 & 9.54 \\
\hline 4 & $11: 55: 28.9$ & $23: 22: 52.7$ & 0.1424 & 42678 & 68 & 5.4 \\
\hline 5 & $11: 55: 27.1$ & $23: 21: 23.1$ & 0.1747 & 52369 & 52 & 6.32 \\
\hline 6 & $11: 55: 26.1$ & $23: 22: 03.5$ & 0.1449 & 43439 & 46 & 9.55 \\
\hline 7 & $11: 55: 26.6$ & $23: 26: 10.2$ & 0.1122 & 33629 & 96 & 2.96 \\
\hline 8 & $11: 55: 23.7$ & $23: 25: 05.9$ & 0.1406 & 42144 & 73 & 4.96 \\
\hline 9 & $11: 55: 22.5$ & $23: 25: 46.3$ & 0.1402 & 42017 & 47 & 7.88 \\
\hline 10 & $11: 55: 20.2$ & $23: 23: 47.3$ & 0.1422 & 42630 & 70 & 5.35 \\
\hline 11 & 11:55:17.5 & $23: 23: 28.8$ & 0.1394 & 41802 & 43 & 10.4 \\
\hline 12 & 11:55:16.7 & $23: 26: 07.8$ & 0.1351 & 40514 & 123 & 2.4 \\
\hline 13 & $11: 55: 15.2$ & $23: 22: 45.8$ & 0.1415 & 42423 & 38 & 11.0 \\
\hline 14 & 11:55:14.1 & $23: 20: 06.1$ & 0.1776 & 53251 & 75 & 3.32 \\
\hline 15 & $11: 55: 14.0$ & $23: 26: 30.8$ & 0.2623 & 78646 & 84 & 2.52 \\
\hline 16 & $11: 55: 13.1$ & $23: 22: 37.6$ & 0.1424 & 42685 & 61 & 6.34 \\
\hline 17 & $11: 55: 12.3$ & $23: 23: 03.9$ & 0.1331 & 39908 & 70 & 5.59 \\
\hline 18 & 11:55:11.3 & $23: 24: 08.1$ & 0.1427 & 42768 & 43 & 8.88 \\
\hline 19 & 11:55:10.1 & $23: 20: 34.6$ & 0.1477 & 44267 & 77 & 3.44 \\
\hline 20 & $11: 55: 08.9$ & $23: 21: 48.4$ & 0.1430 & 42874 & 52 & 7.43 \\
\hline 21 & $11: 55: 09.0$ & $23: 26: 23.5$ & 0.1441 & 43203 & 41 & 9.38 \\
\hline 22 & 11:55:07.9 & $23: 25: 21.2$ & 0.1401 & 41994 & 53 & 6.81 \\
\hline 23 & $11: 55: 05.1$ & $23: 24: 21.3$ & 0.1360 & 40775 & 42 & 9.46 \\
\hline 24 & $11: 55: 01.0$ & $23: 21: 47.3$ & 0.1331 & 39896 & 55 & 6.77 \\
\hline 25 & 11:55:00.6 & $23: 22: 18.9$ & 0.1109 & 33234 & 78 & 4.44 \\
\hline 26 & 11:55:00.2 & $23: 25: 40.2$ & 0.1159 & 34741 & 99 & 2.78 \\
\hline 27 & 11:54:58.6 & $23: 25: 23.6$ & 0.1334 & 39978 & 34 & 11.7 \\
\hline 28 & $11: 55: 35.1$ & $23: 23: 48.4$ & 0.1431 & 42896 & 53 & 7.75 \\
\hline 29 & $11: 55: 27.1$ & $23: 28: 19.3$ & 0.1492 & 44743 & 59 & 6.07 \\
\hline 30 & $11: 55: 25.9$ & $23: 22: 50.9$ & 0.1316 & 39465 & 66 & 3.36 \\
\hline 31 & $11: 55: 22.6$ & $23: 24: 11.3$ & 0.1438 & 43112 & 69 & 5.6 \\
\hline 32 & $11: 55: 21.8$ & $23: 24: 00.1$ & 0.1462 & 43829 & 100 & 2.92 \\
\hline 33 & 11:55:18.6 & $23: 24: 24.0$ & 0.1379 & 41335 & 83 & 4.05 \\
\hline 34 & 11:55:17.6 & $23: 22: 54.9$ & 0.1323 & 39660 & 134 & 2.64 \\
\hline 35 & $11: 55: 17.7$ & $23: 27: 39.8$ & 0.1310 & 39273 & 75 & 4.32 \\
\hline 36 & 11:55:16.7 & $23: 20: 40.9$ & 0.1449 & 43435 & 64 & 4.83 \\
\hline 37 & $11: 55: 17.1$ & $23: 23: 52.6$ & 0.1369 & 41042 & 54 & 7.11 \\
\hline 38 & $11: 55: 15.7$ & $23: 24: 00.6$ & 0.1449 & 43428 & 61 & 5.89 \\
\hline 39 & $11: 55: 15.8$ & $23: 27: 51.6$ & 0.1434 & 42984 & 62 & 4.72 \\
\hline 40 & $11: 55: 15.1$ & $23: 27: 54.4$ & 0.1522 & 45625 & 60 & 5.54 \\
\hline 41 & 11:55:11.4 & $23: 26: 18.7$ & 0.1470 & 44080 & 47 & 8.11 \\
\hline 42 & 11:55:09.0 & $23: 27: 45.4$ & 0.1335 & 40027 & 89 & 3.89 \\
\hline 43 & $11: 55: 03.4$ & $23: 22: 01.8$ & 0.1441 & 43213 & 48 & 7.94 \\
\hline 44 & $11: 55: 35.6$ & $23: 24: 38.7$ & 0.1508 & 45200 & 70 & 5.74 \\
\hline 45 & $11: 55: 34.4$ & $23: 21: 04.2$ & 0.1457 & 43672 & 76 & 3.39 \\
\hline 46 & $11: 55: 34.2$ & $23: 25: 01.8$ & 0.1112 & 33326 & 43 & 7.34 \\
\hline 47 & $11: 55: 33.0$ & $23: 20: 30.8$ & 0.1415 & 42430 & 65 & 4.04 \\
\hline 48 & $11: 55: 32.0$ & $23: 26: 44.3$ & 0.1361 & 40787 & 50 & 6.43 \\
\hline 49 & $11: 55: 30.8$ & $23: 22: 05.3$ & 0.1410 & 42269 & 92 & 2.33 \\
\hline 50 & $11: 55: 29.6$ & $23: 21: 57.7$ & 0.1455 & 43610 & 94 & 2.91 \\
\hline 51 & $11: 55: 26.6$ & 23:27:09.1 & 0.1403 & 42048 & 92 & 3.41 \\
\hline 52 & $11: 55: 25.9$ & $23: 27: 47.0$ & 0.1381 & 41414 & 43 & 8.06 \\
\hline 53 & $11: 55: 23.0$ & $23: 24: 25.5$ & 0.1379 & 41329 & 80 & 4.31 \\
\hline 54 & 11:55:19.0 & $23: 21: 00.1$ & 0.1457 & 43671 & 45 & 8.47 \\
\hline 55 & $11: 55: 19.2$ & $23: 24: 16.5$ & 0.1433 & 42955 & 133 & 2.94 \\
\hline 56 & $11: 55: 18.4$ & $23: 23: 40.8$ & 0.1455 & 43609 & 36 & 11.5 \\
\hline 57 & 11:55:18.1 & $23: 26: 28.4$ & 0.1428 & 42805 & 44 & 9.76 \\
\hline 58 & $11: 55: 17.2$ & $23: 23: 15.6$ & 0.1441 & 43201 & 78 & 3.45 \\
\hline
\end{tabular}

Notes. The data are centered on the cluster Abell 1413. $R$ is the correlation peak as defined by Tonry \& Davis (1979). 
Table A.1. continued.

\begin{tabular}{|c|c|c|c|c|c|c|}
\hline ID & $\begin{array}{c}\text { RA } \\
\text { J2000 }\end{array}$ & $\begin{array}{c}\text { Dec } \\
\text { J2000 }\end{array}$ & $\begin{array}{c}\text { Redshift } \\
z\end{array}$ & $\begin{array}{l}\text { Velocity } \\
\left(\mathrm{km} \mathrm{s}^{-1}\right)\end{array}$ & $\begin{array}{c}\text { Error } \\
\left(\mathrm{km} \mathrm{s}^{-1}\right)\end{array}$ & $R$ \\
\hline 59 & $11: 55: 17.7$ & $23: 27: 57.8$ & 0.1404 & 42084 & 41 & 9.19 \\
\hline 60 & $11: 55: 16.3$ & $23: 25: 21.7$ & 0.1485 & 44504 & 43 & 9.13 \\
\hline 61 & $11: 55: 15.0$ & $23: 26: 56.7$ & 0.1460 & 43770 & 78 & 2.94 \\
\hline 62 & $11: 55: 12.3$ & $23: 20: 57.9$ & 0.0865 & 25932 & 79 & 3.0 \\
\hline 63 & 11:55:09.7 & $23: 23: 46.5$ & 0.1384 & 41477 & 105 & 2.78 \\
\hline 64 & $11: 55: 08.9$ & $23: 21: 24.8$ & 0.1048 & 31413 & 101 & 2.81 \\
\hline 65 & $11: 55: 07.0$ & $23: 24: 20.0$ & 0.1424 & 42681 & 77 & 5.37 \\
\hline 66 & $11: 55: 04.7$ & $23: 21: 13.3$ & 0.1450 & 43465 & 56 & 5.75 \\
\hline 67 & $11: 55: 03.7$ & $23: 24: 47.3$ & 0.1416 & 42456 & 78 & 4.58 \\
\hline 68 & $11: 55: 03.2$ & $23: 20: 57.3$ & 0.1053 & 31562 & 86 & 2.93 \\
\hline 69 & $11: 55: 02.4$ & $23: 26: 30.4$ & 0.1435 & 43031 & 85 & 3.0 \\
\hline 70 & $11: 55: 32.5$ & $23: 19: 14.3$ & 0.1326 & 39763 & 139 & 3.79 \\
\hline 71 & $11: 55: 30.5$ & $23: 16: 48.0$ & 0.0525 & 15726 & 20 & 0.0 \\
\hline 72 & $11: 55: 29.7$ & $23: 20: 16.8$ & 0.0524 & 15714 & 60 & 0.0 \\
\hline 73 & $11: 55: 27.7$ & $23: 17: 41.7$ & 0.1387 & 41584 & 60 & 7.51 \\
\hline 74 & $11: 55: 26.7$ & $23: 15: 47.2$ & 0.1400 & 41959 & 85 & 4.62 \\
\hline 75 & $11: 55: 26.8$ & $23: 20: 00.3$ & 0.1439 & 43145 & 102 & 4.03 \\
\hline 76 & $11: 55: 25.6$ & $23: 19: 38.3$ & 0.1756 & 52645 & 75 & 5.31 \\
\hline 77 & $11: 55: 24.7$ & $23: 19: 28.5$ & 0.1713 & 51369 & 70 & 5.1 \\
\hline 78 & $11: 55: 24.1$ & $23: 15: 50.0$ & 0.0999 & 29953 & 60 & 6.83 \\
\hline 79 & $11: 55: 21.9$ & $23: 15: 26.8$ & 0.1000 & 29981 & 40 & 10.5 \\
\hline 80 & $11: 55: 22.0$ & $23: 21: 47.7$ & 0.1456 & 43661 & 91 & 4.26 \\
\hline 81 & $11: 55: 21.2$ & $23: 16: 10.7$ & 0.1356 & 40660 & 20 & 0.0 \\
\hline 82 & $11: 55: 21.0$ & $23: 19: 32.6$ & 0.1743 & 52264 & 79 & 4.67 \\
\hline 83 & $11: 55: 20.1$ & $23: 19: 12.9$ & 0.1428 & 42806 & 82 & 4.25 \\
\hline 84 & $11: 55: 19.7$ & $23: 20: 59.8$ & 0.1454 & 43600 & 78 & 4.96 \\
\hline 85 & 11:55:18.8 & $23: 21: 31.8$ & 0.1500 & 44977 & 55 & 6.09 \\
\hline 86 & $11: 55: 17.6$ & $23: 19: 15.0$ & 0.1419 & 42545 & 104 & 3.96 \\
\hline 87 & $11: 55: 17.2$ & $23: 21: 50.5$ & 0.1372 & 41123 & 107 & 4.42 \\
\hline 88 & 11:55:16.3 & $23: 21: 46.3$ & 0.2883 & 86430 & 53 & 5.86 \\
\hline 89 & $11: 55: 15.0$ & $23: 20: 21.2$ & 0.1729 & 51819 & 66 & 5.4 \\
\hline 90 & $11: 55: 12.2$ & $23: 18: 51.6$ & 0.1419 & 42530 & 106 & 3.79 \\
\hline 91 & 11:55:10.6 & $23: 16: 19.2$ & 0.0644 & 19301 & 152 & 2.75 \\
\hline 92 & $11: 55: 11.0$ & $23: 19: 36.5$ & 0.1391 & 41696 & 70 & 6.53 \\
\hline 93 & 11:55:09.1 & $23: 17: 42.3$ & 0.1444 & 43275 & 92 & 4.0 \\
\hline 94 & 11:55:08.4 & $23: 21: 08.5$ & 0.1423 & 42664 & 48 & 8.04 \\
\hline 95 & 11:55:07.1 & $23: 17: 24.9$ & 0.1390 & 41685 & 60 & 4.66 \\
\hline 96 & $11: 55: 35.0$ & $23: 29: 53.9$ & 0.0171 & 5130 & 30 & 0.0 \\
\hline 97 & $11: 55: 29.4$ & $23: 33: 12.2$ & 0.1415 & 42420 & 270 & 2.6 \\
\hline 98 & $11: 55: 23.6$ & $23: 31: 50.7$ & 0.1439 & 43125 & 80 & 4.51 \\
\hline 99 & $11: 55: 16.7$ & $23: 28: 49.0$ & 0.1414 & 42388 & 79 & 4.05 \\
\hline 100 & 11:55:13.1 & $23: 33: 26.1$ & 0.1388 & 41623 & 68 & 4.5 \\
\hline 101 & $11: 55: 10.3$ & $23: 31: 36.9$ & 0.2387 & 71567 & 105 & 2.77 \\
\hline 102 & 11:55:07.7 & $23: 32: 21.9$ & 0.1494 & 44774 & 71 & 5.78 \\
\hline 103 & 11:54:59.4 & $23: 31: 23.0$ & 0.1396 & 41861 & 93 & 3.26 \\
\hline 104 & $11: 55: 29.3$ & $23: 20: 13.7$ & 0.0525 & 15730 & 20 & 0.0 \\
\hline 105 & $11: 55: 33.5$ & $23: 24: 58.6$ & 0.1383 & 41453 & 49 & 8.55 \\
\hline 106 & $11: 55: 16.6$ & $23: 23: 17.4$ & 0.1419 & 42541 & 87 & 4.45 \\
\hline 107 & $11: 54: 57.4$ & $23: 24: 03.8$ & 0.1346 & 40350 & 58 & 4.3 \\
\hline
\end{tabular}

